\title{
MULTIPLE FAILURE SURVIVABILITY IN WDM NETWORKS WITH $p$-CYCLES
}

\author{
D.A. Schupke \\ Munich University of Technology \\ Institute of Communication Networks \\ D-80290 Munich, Germany
}

\begin{abstract}
Preconfigured protection cycles or " $p$-cycles" can attain high capacity efficiency and fast protection switching times when deployed in WDM mesh networks. We consider the survivability of $p$-cycles in presence of multiple failures which can occur in larger networks.
\end{abstract}

\section{INTRODUCTION}

p-Cycles are attractive for WDM network protection, since high capacity efficiency and fast protection switching times can be achieved $[1,2,3]$. WDM $p$-cycles guarantee to survive any single fiber duct failure, e.g., caused by a backhoe. But for larger networks dual failures - though less probable than single failures-should be taken into account [4, 5]. Thus, the behavior of $p$-cycles in presence of dual fiber duct failures is important. Because of very low probability, failure events of order higher than two can be neglected for WDM networks [5]. Also, WDM nodes can often be regarded as very reliable, since, e.g., internal redundancy is used.

Fig. 1 (a) depicts a network with one $p$-cycle (A-B-D-CA). This $p$-cycle is able to protect on-cycle links as shown in Fig. 1 (b) for the on-cycle link A-B. Furthermore, a $p$ cycle is able to protect straddling links. A straddling link is an off-cycle link having $p$-cycle nodes as endpoints. In the case of a straddling link failure, each $p$-cycle can protect two working capacity units on the link by providing the two alternative paths around the $p$-cycle as shown in Fig. 1 (c) for the failure of straddling link B-C. For both on-cycle and straddling links, the protection switching can be made very fast, since the nodes neighboring the failure need to perform any real-time actions.

In a larger network, multiple $p$-cycles can be deployed [1, 3]. The general problem for a two-connected and capacitated network seeks an optimal routing for the demands and an optimal configuration of $p$-cycles protecting

D.A. Schupke is supported by the German Federal Ministry of Education and Research, project TransiNet. Email: Schupke@1kn.ei.tum.de. these. This problem can be solved jointly [6], or by a twostep approach where first the demand connections are routed through the network and then the $p$-cycles are formed $[1,3]$.

The routed demands reserve working capacity in the network. The spare capacity is the remaining available capacity, in which the $p$-cycles are formed as protection capacity. The set of $p$-cycles is chosen such that for every duct the working connections are protected by $p$-cycles of corresponding capacity. By its protection paths, a $p$-cycle capacity unit covers one working capacity unit per on-cycle link, and two units per straddling link.

After successful $p$-cycle assignment, the network is guaranteed to be protected against single fiber duct failures. Subsequent failures in presence of a single failure can be survived by finding $p$-cycles in the remaining topology, i.e. the topology without the failed element [7]. An example is given in Fig. 2 (a)-(b). For this approach, the $p$-cycles have to be reconfigured based on the new topology and the new paths (which have been restored). As protection capacity is reconfigured, it is non-disruptive for the working paths. A successful reconfiguration protecting all secondary failures requires enough spare capacity and connectivity.

But what if, after a first failure, the $p$-cycles remain as initially configured? This can happen in these cases:

- Reconfigurations are not desired, e.g., adaptive changes of the protection configuration should be avoided.

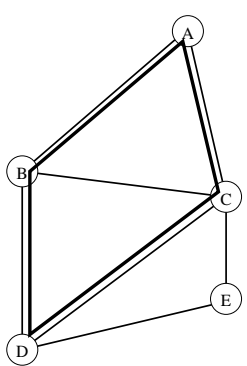

(a)

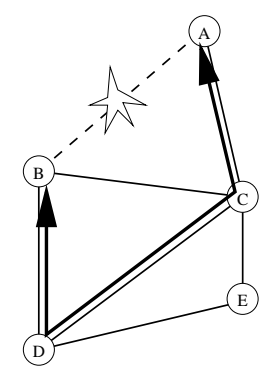

(b)

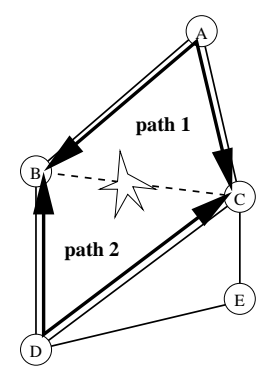

(c)
Figure 1: A network with one $p$-cycle (a) which can protect on-cycle links (b) and straddling links (c). 
- A reconfiguration is not possible, e.g., in a fixed $p$ cycle infrastructure [1] or due to lack of capacity.

- The reconfiguration after a first failure is not completed.

In this context we consider how such "static" $p$-cycles perform upon dual fiber duct failures, and how we can improve the performance by modifying the selection of $p$-cycles. The next section considers double duct failures in (static) $p$-cycle networks, for which we investigate a case study of a pan-European network in Section 3. Section 4 concludes this paper.

\section{STATIC $p$-CYCLES AND DUAL DUCT FAILURES}

In this section we overview which dual duct failures cannot be survived. A detailed analysis can be found in [8]. We assume that ducts fail independently, i.e. the ducts are mutually disjoint (enough) such that a single failure intrusion does not affect multiple ducts.

If the network is protected by multiple $p$-cycles and a double failure does not occur twice in any $p$-cycle, the individual failures are guaranteed to survive by separate $p$-cycles, since each cycle "sees" just a single failure (Fig. 2 (c)). Consequently, a double failure analysis needs only to consider occurrences of double failures within a cycle.

Fig. 3 depicts the results of a dual failure analysis within a single $p$-cycle. Only those failure situations are shown which cause loss of protected working units. The number of lost connections can be lower, since two lost working capacity units can belong to the same connection.

For some entries in the matrix two different loss values can occur, since the loss is dependent on the protection paths used. Obviously, double failures have to be treated as ordered events (failure at time $t_{1}$, failure at time $t_{2}$ ) with $t_{1}<t_{2}$, since the traffic loss can be dependent on the sequence of failures. Note that, because of the failure inde-

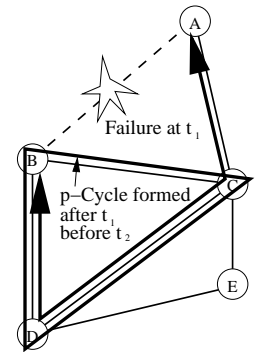

(a)

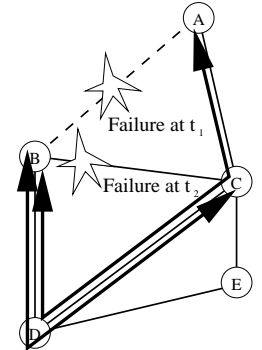

(b)

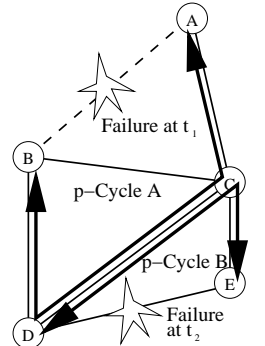

(c)
Figure 2: In (a), a new $p$-cycle is formed after a first (oncycle) failure which can protect a subsequent failure during the repair of the first failure (b). Two failures affecting two $p$-cycles independently (c).

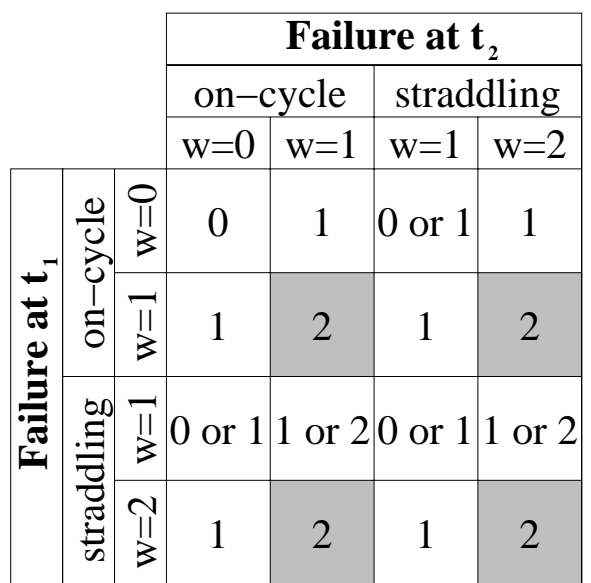

Figure 3: The number of lost working capacity units by double failures in dependence on the protected working capacity $w$ of on-cycle and straddling links [8]. The shaded entries correspond to fully loaded links. Note that a protection switching is ineffective if the protection path is already (partially) used.

pendence and the fast recovery time of $p$-cycles, dual failure scenarios with $t_{2}<t_{1}+t_{\text {recover }}$ are negligible.

The protection switching analyzed is based on link information. If nodes also take demand information into account, a better double failure survivability can be achieved. Fig. 4 (a) depicts the situation where a $p$-cycle recovers a single duct failure C-D for a demand path A-B-C-D. A second failure $\mathrm{B}-\mathrm{C}$ on the demand path can be survived, if after the second failure node $\mathrm{B}$ switches to the active $p$-cycle. It is worth mentioning that in this situation, the second failure cannot be survived by $p$-cycle reconfiguration (before the second failure) only.

For the switching at $\mathrm{B}$, a node requires the knowledge about the association of the demand paths to the $p$-cycles, and not just the demand-to-link relationships. Moreover, the information that an active $p$-cycle protects a secondly failed link with the same demand is required to avoid demand path misconfigurations. This can be signaled from the switching nodes of the first failure. In the example, node D can notify the nodes along the cycle (including B). Note that a node failure, e.g. node $\mathrm{C}$, can be survived similarly.

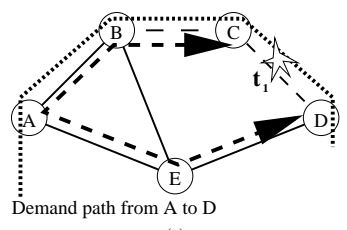

(a)

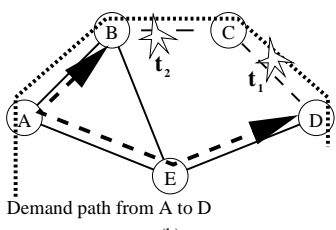

(b)
Figure 4: A protection switching of $\mathrm{C}$ and $\mathrm{D}$ after a first failure on C-D (a), and a subsequent switching of B after a secondary failure on B-C (b). Thus B-A-E-D replaces B-C$\mathrm{D}$, a subpath instead of a link. 


\section{CASE STUDY}

In this case study (see also [8]), we optimize the panEuropean COST 239 network (Fig. 5) consisting of 11 nodes with full wavelength conversion and 26 ducts [3]. We use a related demand matrix offering 1760 bidirectional lightpath demands to the network. The network has a high average degree of 4.7, thus, it can have high double failure survivability. The number of fibers per duct is two and the number of wavelengths of each fiber is 128 .

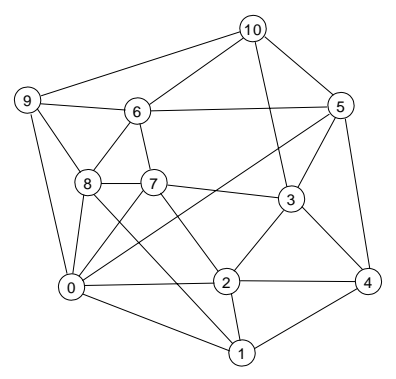

Figure 5: Nodes and ducts of the COST 239 network.

A lower bound for the efficiency, the protection to working capacity ratio, of a link restoration mechanism protecting single failures [9] and dual failures can be developed for an idealized situation. Assume the capacity on the ducts at the restoration paths' end-nodes are the main bottleneck [9]. Hence, path blocking does not occur within the network, but rather due to insufficient spare capacity at the end-nodes of failed spans.

From this point-of-view, among the $f$-failures we consider the situations where $f$ ducts incident to a single node $n$ of degree $d$ are hit $(f<d)$. To generate a lower bound on the spare capacity, we assume each adjacent duct of such a node has the same amount of working capacity $w$. That way, after any failure of $f$ incident ducts, each surviving adjacent duct provides a spare capacity of $\frac{f w}{d_{n}-f}$ at minimum.

Thus, the efficiency per duct calculates to $\frac{f}{d_{n}-f}$. This can be generalized ${ }^{1}$ from the specific end-node to a network-wide lower bound of $e_{f}=\frac{f}{\bar{d}-f}$, with $\bar{d}$ the average degree. We obtain $e_{2}=74 \%$ as efficiency lower bound for dual failures in the COST 239 network.

The $p$-cycles are configured according to the two-step approach (working connections are routed on the minimum hop path, see Sec. 1) and with different objectives:

1. $P$ : Minimize the protection capacity.

2. $N$ : Minimize the number of selected cycles.

3. $-N$ : Maximize the number of selected cycles.

4. $W$ : Minimize working capacity coverage of the $p$ cycle (among the selected ones) with the maximum

\footnotetext{
${ }^{1}$ Using (I) the average spare capacity on a duct is less than or equal to the maximum spare capacity on a duct, and (II) the harmonic mean is less than or equal to the arithmetic mean.
}

working capacity coverage. This minimax objective tries to keep the dual failure susceptibility of the $p$ cycles low (the higher a $p$-cycle's working capacity, the more failures to protect).

We develop an upper bound measure for the loss caused by a double fiber duct failure by counting:

i) all working links which are affected by the first failure and whose $p$-cycle is affected by the second failure

ii) all working links which are affected by the second failure and whose $p$-cycle is already used.

The shaded entries in Fig. 3 are the cycle's loss-values for failed link-pairs with maximum working coverage. Note that in these capacity-optimal cases (among other cases) the upper bound on the loss is tight.

Based on this loss calculation we calculate $\bar{R}$, the average restorability of affected working traffic. The restorability $R_{(a, b)}$ of a double failure $(a, b)$ is defined in this paper (similar to [5]) as the portion of all working capacity units $w_{a}+w_{b}$ on the ducts $a$ and $b$ that are simultaneously affected by a double failure and survive this failure. This can be expressed using the complentary portion by:

$$
R_{(a, b)}=1-\frac{\operatorname{Loss}(a, b)}{w_{i}+w_{j}}
$$

$\bar{R}$ is then the average of $R_{(a, b)}$ over all double failure events.

The mean restorability (Fig. 6) for the capacity-optimal configuration $(P)$ achieves on average 57\% only, which motivates to take dual failures into account when designing a $p$-cycle network. The dependence on the number of cycles is also visible by the bounding values: The configurations with minimum $(N)$ and maximum $(-N)$ number of cycles attain restorability averages of $41 \%$ and $71 \%$, respectively. A larger number of cycles means a larger number of cycle pairs which are able to protect dual failures (Sec. 2). The minimax working capacity coverage optimization $(W)$ reaches an average of $76 \%$, the best restorability level.

The configuration with the maximum number of cycles $(-N)$ has some hundreds of $p$-cycles, which is impractical for the network configuration. But it shows that just taking many cycles cannot always aid in attaining high dual failure survivability. The capacity-optimal $(P)$ and minimum-cycle-number $(N)$ configurations require two orders of magnitude less cycles. Some ten $p$-cycles suffice for the minimax working capacity coverage optimization $(W)$.

Fig. 7 shows the efficiency over the allowed maximum physical $p$-cycle length. The capacity $(P)$ and least-cycles $(N)$ optimizations are very capacity efficient with values of $48 \%$ and $58 \%$, respectively. However, both stay below the lower bound $e_{2}$ and thus, are weak in restorability (Fig. 6). Using the maximal number of cycles $(-N)$ consumes more than an additional of $124 \%$ working capacity for protection. The minimax working capacity coverage optimization $(W)$ 


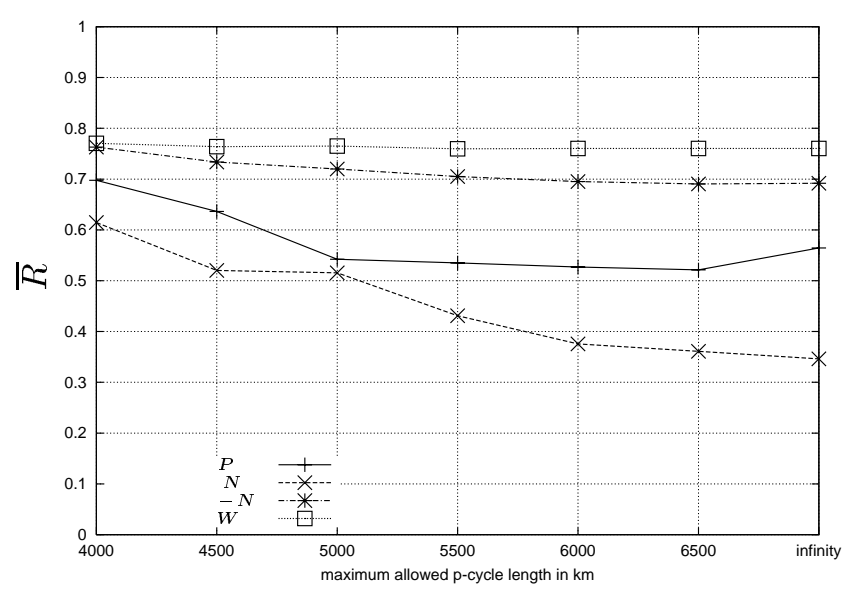

Figure 6: The mean restorability of a double failure event over the allowed maximum physical $p$-cycle length.

is with an average of $65 \%$ efficient, and comes close to the lower bound $e_{2}=74 \%$. It combines high restorability with efficiency.

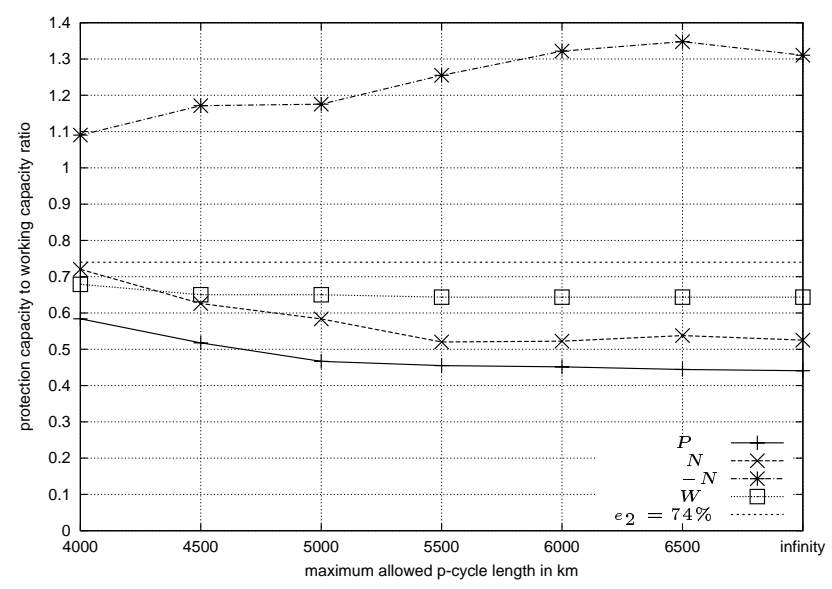

Figure 7: The efficiency ratio over the allowed maximum physical $p$-cycle length.

\section{CONCLUSIONS}

This paper brings $p$-cycles in WDM networks and multiple failure survivability together. Link $p$-cycles protect single fiber duct failures. Multiple failures can be survived, if each failure is on a different $p$-cycle. Multiple failures can also be survived using cycle reconfiguration, which is hitless for working traffic. Special multiple failures (also node failures) on a single $p$-cycle can be recovered by failure signaling, even if reconfiguration is ineffective. Finally, this paper presents the loss values in a single $p$-cycle depending on the working capacity of the protected links.

For evaluation, we developed a measure approximating the loss and the restorability upon double failures. In a case study with $p$-cycle designs for a pan-European network, we obtained the following averaged results using different design objectives:

- The capacity optimal and the minimal number of cycles designs are only able to restore around a half of the connections after dual failures. The efficiency of the latter is comparable to the former.

- When maximizing the number of cycles, we obtain higher dual failure restorability. However, this can require a huge amount of cycles and high protection capacity.

- Minimizing the maximum working capacity coverage of selected $p$-cycles is promising to achieve high dual failure restorability (about 3/4 of affected connections). Capacity is consumed efficiently and a reasonable number of cycles is required.

\section{REFERENCES}

[1] W.D. Grover and D. Stamatelakis, "Bridging the ringmesh dichotomy with p-cycles," in Proc. of DRCN Workshop, 2000.

[2] W. Grover, J. Doucette, M. Clouqueur, D. Leung, and D. Stamatelakis, "New options and insights for survivable transport networks," IEEE Comm. Mag., vol. 40, no. 1, Jan 2002.

[3] D.A. Schupke, C.G. Gruber, and A. Autenrieth, "Optimal Configuration of $p$-Cycles in WDM Networks," in Proc. of IEEE ICC, 2002.

[4] D.A. Schupke, A. Autenrieth, and T. Fischer, "Survivability of Multiple Fiber Duct Failures," in Proc. of DRCN Workshop, 2001.

[5] M. Clouqueur and W.D. Grover, "Dual failure availability analysis of span-restorable mesh networks," IEEE JSAC, vol. 20, no. 4, May 2002.

[6] W.D. Grover and J.E. Doucette, "Advances in Optical Network Design with p-Cycles: Joint optimization and pre-selection of candidate p-cycles," in Proc. of IEEELEOS Summer Topical Meeting, 2002.

[7] C.G. Gruber and D.A. Schupke, "Capacity-efficient Planning of Resilient Networks with $p$-Cycles," in Proc. of Networks, 2002.

[8] D.A. Schupke, "The Tradeoff Between the Number of Deployed $p$-Cycles and the Survivability to Dual Fiber Duct Failures," in Proc. of IEEE ICC, 2003.

[9] J. Doucette and W.D. Grover, "Comparison of Mesh Protection and Restoration Schemes and the Dependency on Graph Connectivity," in Proc. of DRCN Workshop, 2001. 